\title{
Ekman Boundary Layers and Energy Dissipation in Rotating Drums During Spin-Down Process
}

\author{
HIROSHI OHUE and GENSHI KAWASHIMA \\ Department of Mechanical Engineering, Musashi Institute of Technology, \\ 1-28-1 Tamazutsumi, Setagaya, Tokyo 158, Japan \\ WEN-JEI YANG \\ Department of Mechanical Engineering and Applied Mechanics, University of Michigan, \\ Ann Arbor, Michigan 48109, USA.
}

(Received August 9, 1995)

\begin{abstract}
The Laser Doppler Velocimetry (LDV) is employed to investigate energy dissipation during a spin-down process inside a rotating drum. The tracer/light sheet method is applied to observe flow patterns in the entire flow field from which the instantaneous, two-dimensional velocity distribution and the formation and subsequent time wise variation of the Ekman boundary layer are determined. Results are synthesized to find the relationship between the Ekman boundary layer and the redistribution of secondary-flow induced angular momentum. The fluid viscosity, drum size and speed of rotation are varied to determine their effects on both the Ekman boundary layer and energy dissipation during spin-down process. The role of Ekman boundary layer in the reduction of rotating fluid motion is determined. Results from the study may be used to develop a method to achieve uniform mixing in an enclosed vessel.
\end{abstract}

Key Words: Spin-down; Ekman boundary layer; Secondary flow; Energy dissipation; Rotating drum

\section{INTRODUCTION}

$\mathrm{R}$ otating machinery is the most important prime mover in power generation and refrigeration cycles. However, recirculating flow in rotating units, for example unsteady flow in a suddenly rotated drum, is complex and is very difficult to visualize or measure. Thus, less effort has been directed to study unsteady flow in a rotating device.

Recently, a series of studies were performed on unsteady transport phenomena in rotating drums subjected to a sudden acceleration or deceleration. These studies include measurements of velocity by means of LDV [1988], hydrodynamic theory [1990a], unsteady recirculating flow with inner surface heating by flow visualization using the laser light sheet method [1990b], flow patterns by means of double-exposure photograph combined with the determination of the temperature measurement by means of thermocouples [1990c, $d$ and 1992a], comparison of results obtained by the laser light sheet and LDV methods [1992b], observation of velocity field in a plane parallel to the axis of a rotating drum [1991], and thermal field visualization by means of microencapsulated temperature-sensitive liquid crystals and image processing [1992c, d].

The purpose of this study is to investigate energy dissipation and formation of the Ekman boundary layer in an unsteady flow during a spin-down process inside a rotating drum by means of both the tracer/light sheet and LDV methods.

$\begin{array}{ll}\text { Nomenclature } & \\ b & : \text { Distance from the midplane } \\ E & : \text { Kinetic energy of fluid in a drum } \\ r & : \text { Radial coordinate of drum } \\ t & : \text { Time } \\ V_{\theta} & : \text { Angular velocity } \\ V_{r} & : \text { Radial velocity } \\ \delta_{E} & : \text { Thickness of Ekman boundary layer }\end{array}$




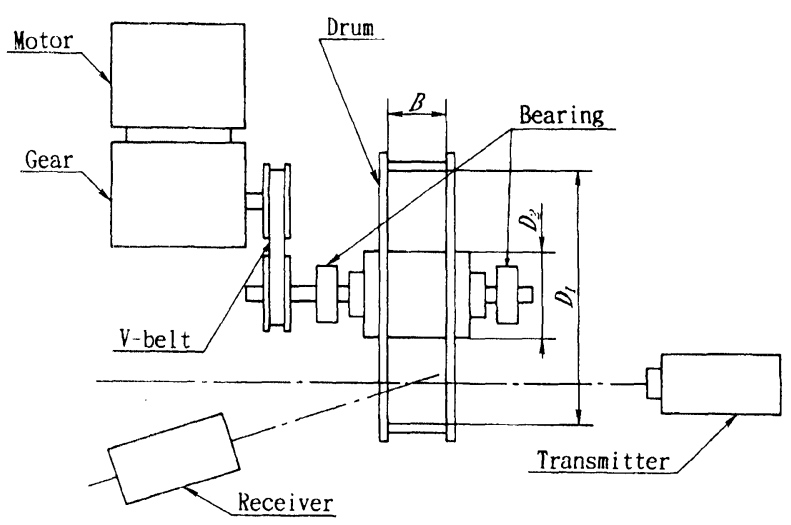

FIGURE 1 A schematic of experimental apparatus.

\section{EXPERIMENTAL APPARATUS}

The experimental apparatus used in this study is illustrated in Fig. 1. Two types of drum were tested whose size is shown in Fig. 2. The drum consisted of an outer cylinder (inside diameter: $D_{1}=240 \mathrm{~mm}$ ), an inner cylinder (outside diameter: $D_{2}=80 \mathrm{~mm}$ ) and two circular plates for the sidewalls $5 \mathrm{~mm}$ in thickness. The widths of the drums were $B=60 \mathrm{~mm}$ (Drum I) and $B=30 \mathrm{~mm}$ (Drum II). The outer cylinder and the two sidewalls were made of transparent material to enable visualization of the flow. These drums were supported by bearings and driven by a variable speed motor via a gear and a V belt. The speeds of rotation were 3 and 5 seconds per revolution. The drums were filled with a water or a glycerin/water solution. The liquid was seeded with light scattering plastic microparticles having a specific weight of 1.02 and a diameter of $150 \mu \mathrm{m}$.

Figure 3 shows the experimental setup for flow visualization. A $16 \mathrm{~mW} \mathrm{He-Ne} \mathrm{laser} \mathrm{was} \mathrm{used} \mathrm{as} \mathrm{a} \mathrm{light}$

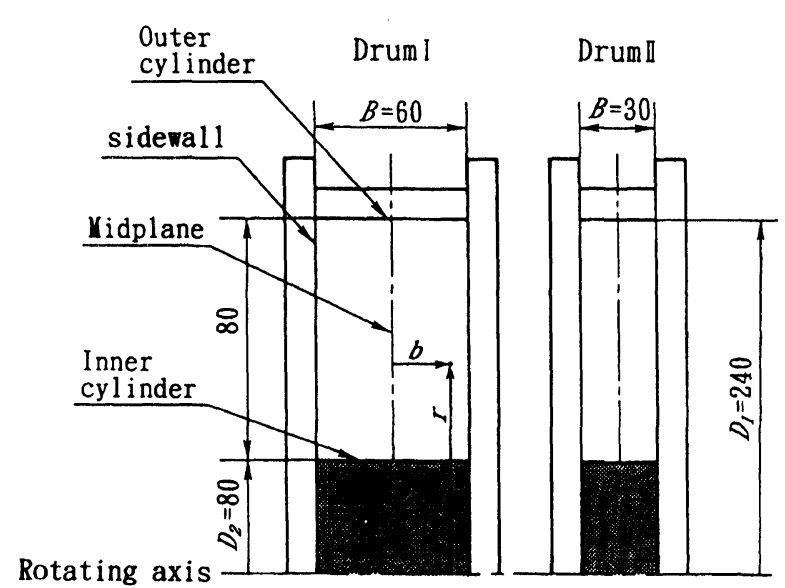

FIGURE 2 Size of drums. source. The light sheet of $1 \mathrm{~mm}$ thick cut the drum normal to the axis. Flow visualization experiments were conducted in a dark room. Flow patterns were observed on an illuminated plane from two directions, perpendicular to and parallel to the rotating axis. Typical photographs at midplane and near the sidewall are shown in Fig. 4. The velocity of each tracer particle was determined from its displacement and direction of its pathline in the photograph. Each trace was separated into two components [1991]: the radial velocity, $V_{r}$, and angular velocity, $V_{\theta}$.

\section{EXPERIMENTAL RESULTS}

In the interest of brevity, only characteristic features of the flow patterns are presented here. Figures 5 and 6 show the angular velocity-time history obtained by LDV. Figure 5 is for Drum II, water, $5 \mathrm{sec} / \mathrm{rev}$ while Fig. 6 is for Drum II, $50 \%$ glycerin/water solution, $5 \mathrm{sec} / \mathrm{rev}$. (a) is at the midplane and (b) is near the side wall. About 0.82 sec elapses before the drum comes to a full stop.

One observes, in Fig. 5(a), a steep dip in the velocity near the surface of the inner cylinder and velocity oscillations near the outer cylinder during initial stage of the transient. The same behavior was observed in Fig. 5(b). In Fig. 6(a), similar velocity oscillations were observed near the outer cylinder but no steep dip in the velocity near the surface of the inner cylinder. Flow oscillations were observed in each measured position in Fig. 6(b).

Next is to consider the kinetic energy of the fluid inside a drum. It is shown in Figs. 5 and 6 that the angular velocity at each measured position in the drum varies in the radial and axial direction. Therefore, a ring

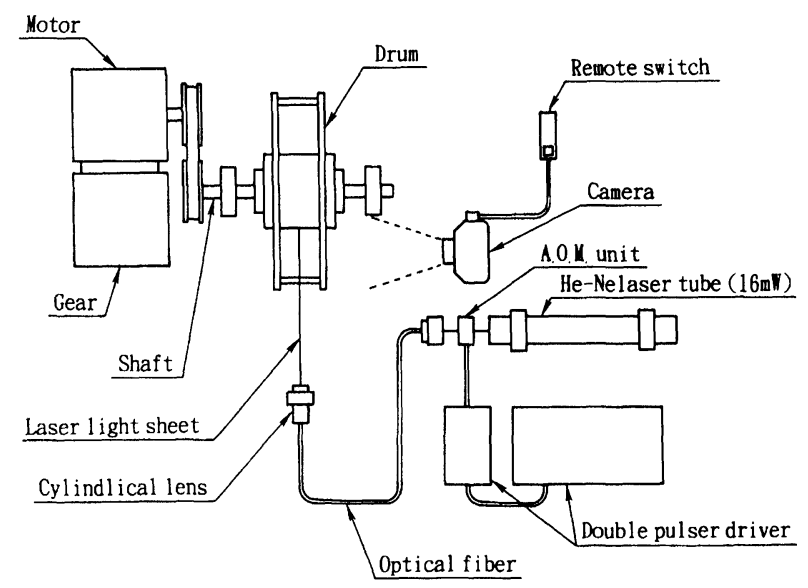

FIGURE 3 A schematic of flow visualization setup. 


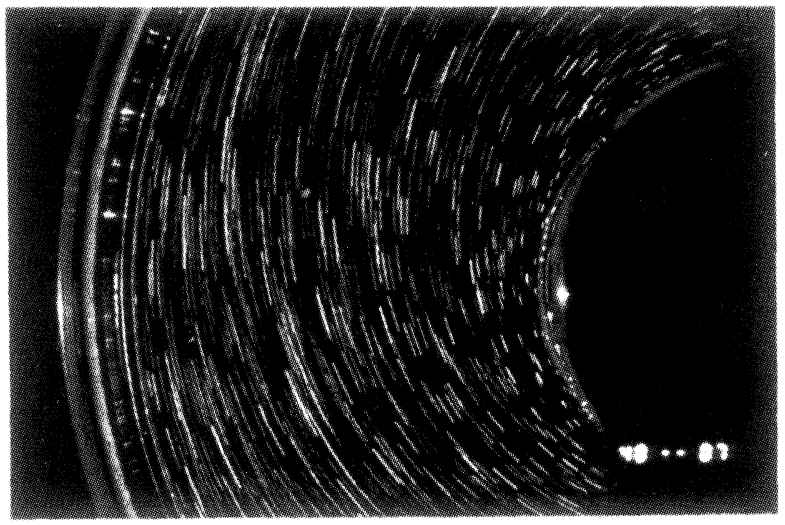

(a) at the midplane

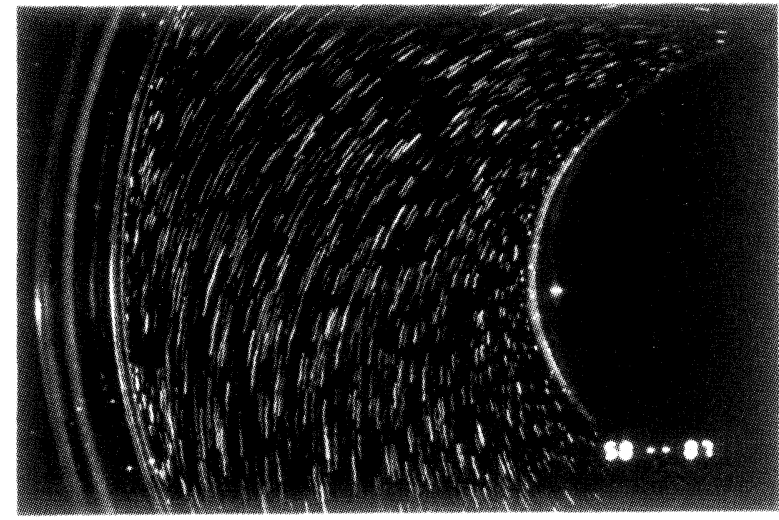

(b) near the sidewall

FIGURE 4 Flow pattern in 50\% glycerin/water solution inside Drum II at 6 sec under a spin-down process from 5 sec/rev to zero. (Flow direction is clockwise).

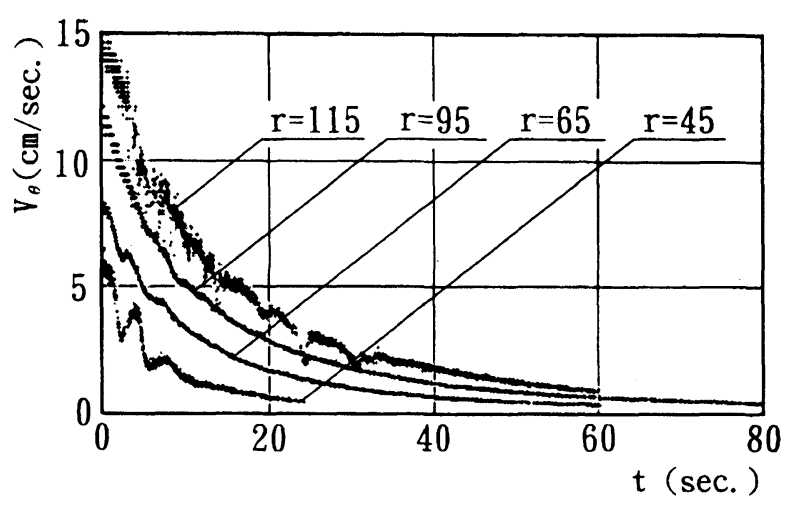

(a) at the midplane

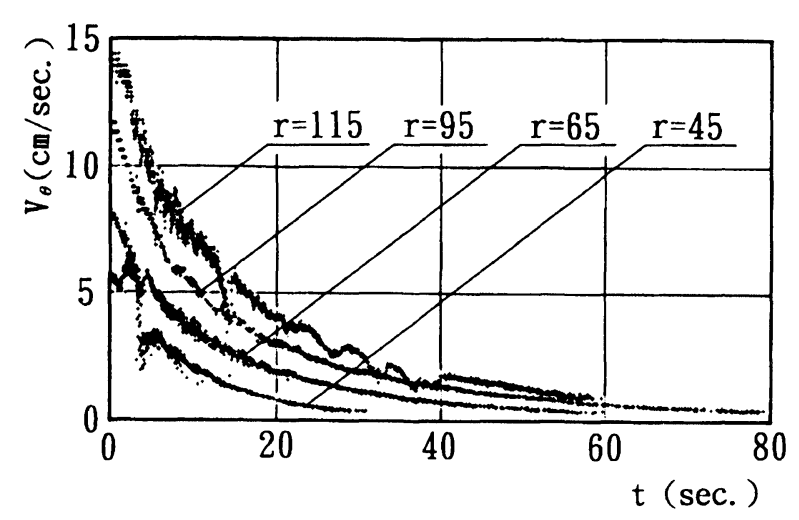

(b) near the sidewall

FIGURE 5 Angular velocity-time history in water inside Drum II under a spin-down process from 5 sec/rev to zero.

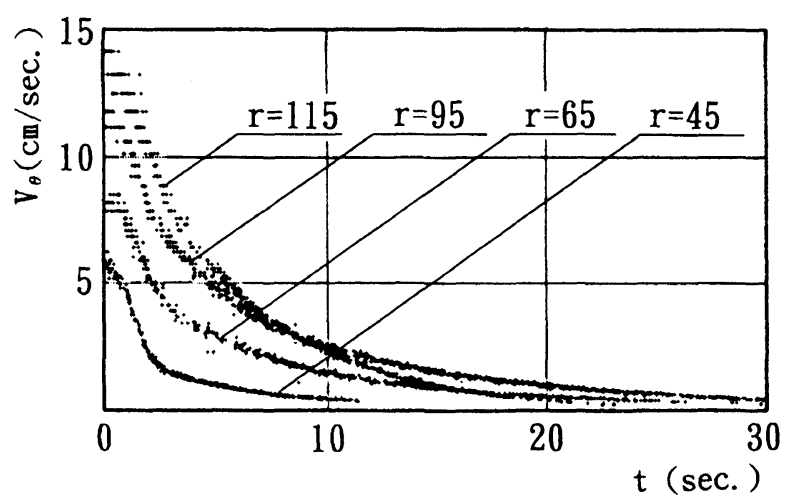

(a) at the midplane

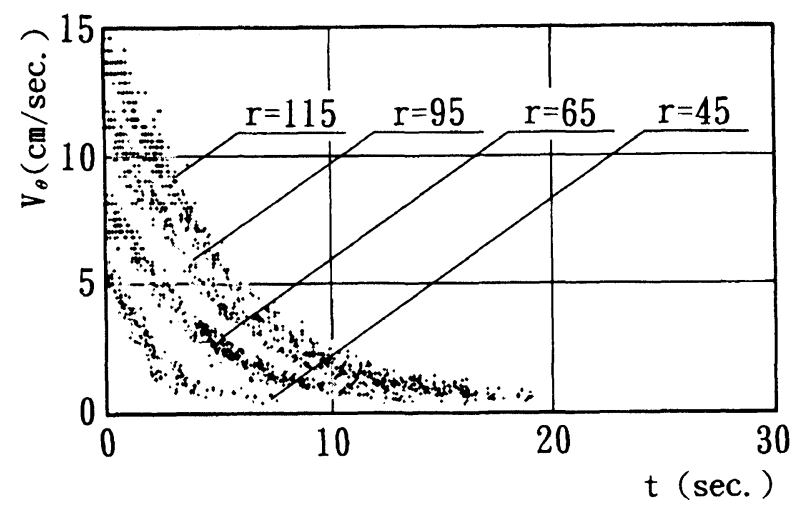

(b) near the sidewall

FIGURE 6 Angular velocity—time history in 50\% glycerin/water solution inside Drum II under a spin-down process from 5 sec/rev to zero. 


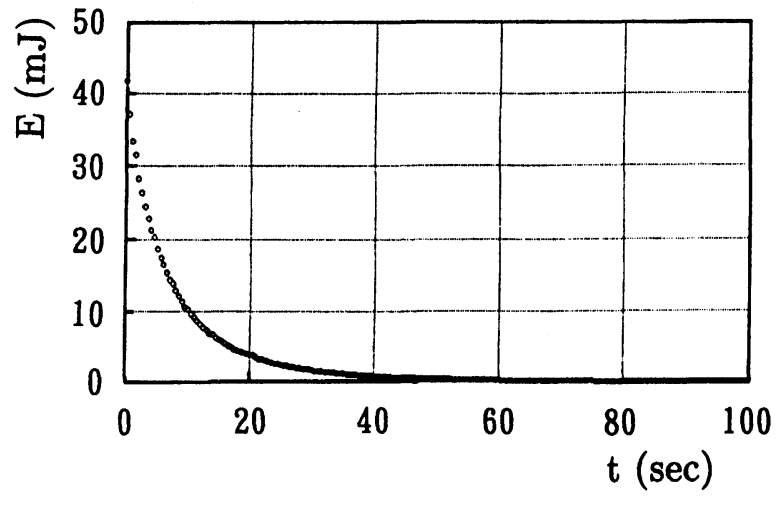

(a) water

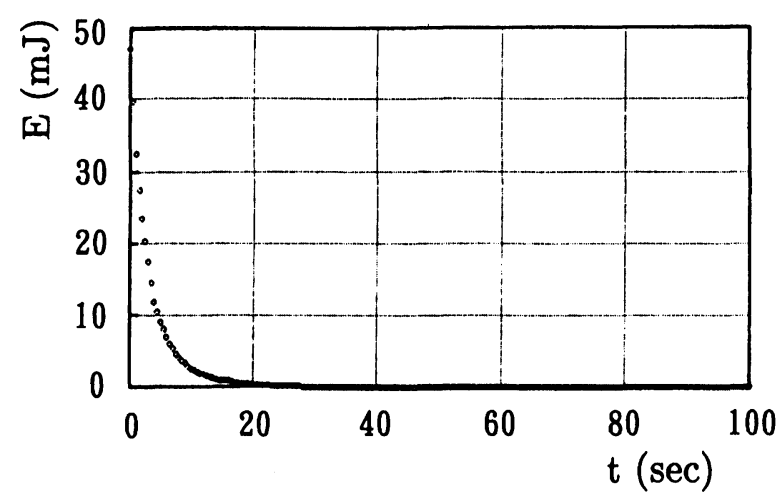

(b) $50 \%$ glycerin/water solution

FIGURE 7 Kinetic energy-time history inside Drum I under a spin-down process from 3 sec/rev to zero.

volume that encompasses the measured position of the angular velocity should be considered and thus the kinetic energy of the fluid in a ring volume was calculated. The total kinetic energy of the fluid in the drum was the sum of the kinetic energy in each ring volume. The angular velocity was measured at every five milli-

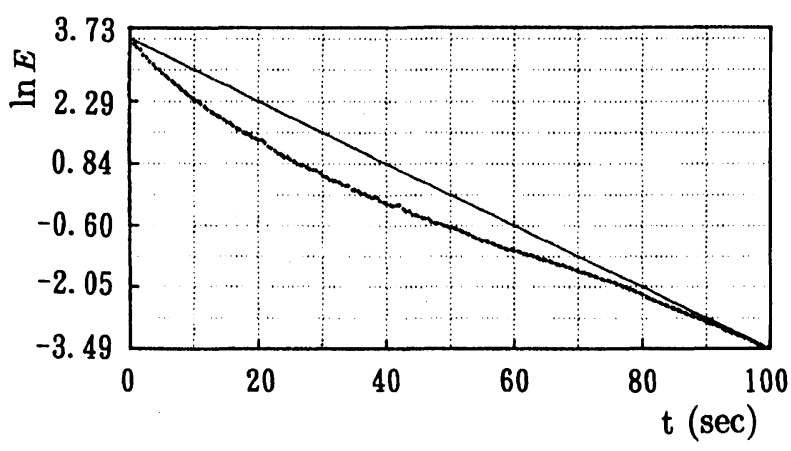

FIGURE 8(a) Logarithmic representation of kinetic energy-time history in water inside Drum I under a spin-down process from 3 sec/rev to zero.

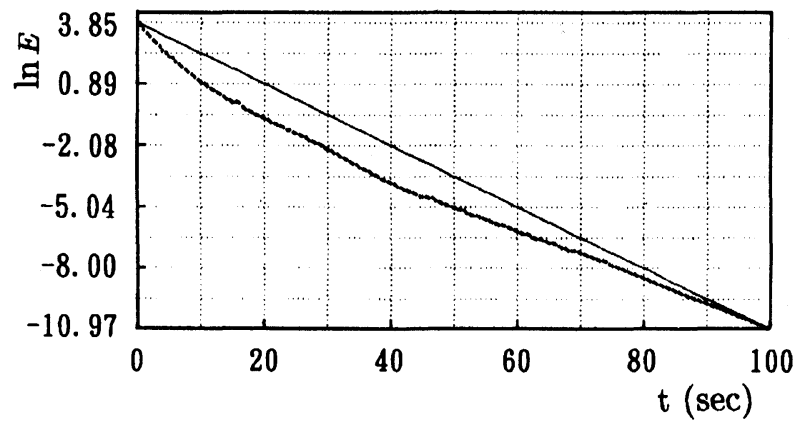

FIGURE 8(b) Logarithmic representation of kinetic energy-time history in 50\% glycerin/water solution inside Drum I under a spindown process from $3 \mathrm{sec} / \mathrm{rev}$ to zero. meter in radial and axial direction. Figure 7 shows the kinetic energy-time history for Drum I, $3 \mathrm{sec} / \mathrm{rev}$, with (a) for water and (b) for $50 \%$ glycerin/water solution. The time needed for the kinetic energy to reach zero from beginning of transient in Fig. 7 (b) was shorter than that in Fig. 7(a).

Figure 8 corresponds to a logarithmic plot of the kinetic energy, (a) for Drum I, water, 3 sec/rev and (b) for Drum I, 50\% glycerin/water solution, $3 \mathrm{sec} / \mathrm{rev}$, corresponding to Figs. 7 (a) and (b), respectively. Figure 8 (c) is for Drum II, $80 \%$ glycerin/water solution, $5 \mathrm{sec} / \mathrm{rev}$. A straight line in these figures indicates an exponential decrease. In other words, a straight line means the dissipation of the total kinetic energy is caused by a decrease in the angular velocity alone. For Drum I, 3 sec/rev in Figs. 8(a) and (b), the deviations between the measured and straight kinetic energy curves are similar. However, in Fig. 8(c) this deviation is very small. For Drum II, $5 \mathrm{sec} / \mathrm{rev}$ case, secondary flow was generated as shown in Figs. 9 and 10. Therefore, the deviation

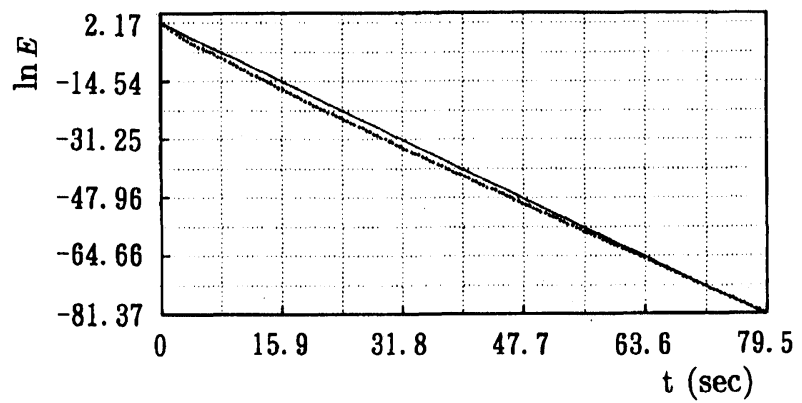

FIGURE 8(c) Logarithmic representation of kinetic energy-time history in $80 \%$ glycerin/water solution inside Drum II under a spindown process from $5 \mathrm{sec} / \mathrm{rev}$ to zero. 


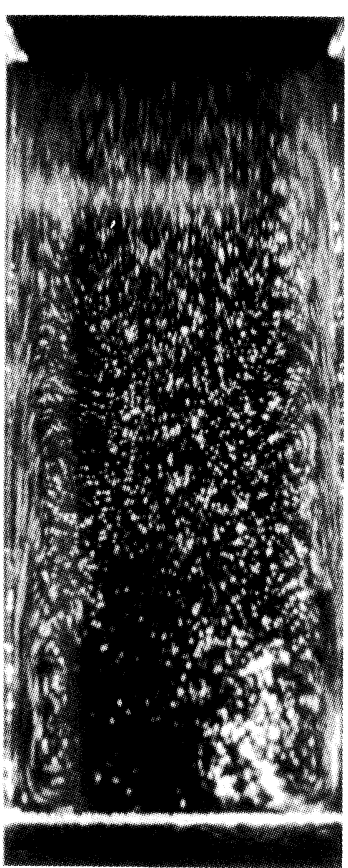

Flow pattern (shutter speed: $1 \mathrm{sec}$ )

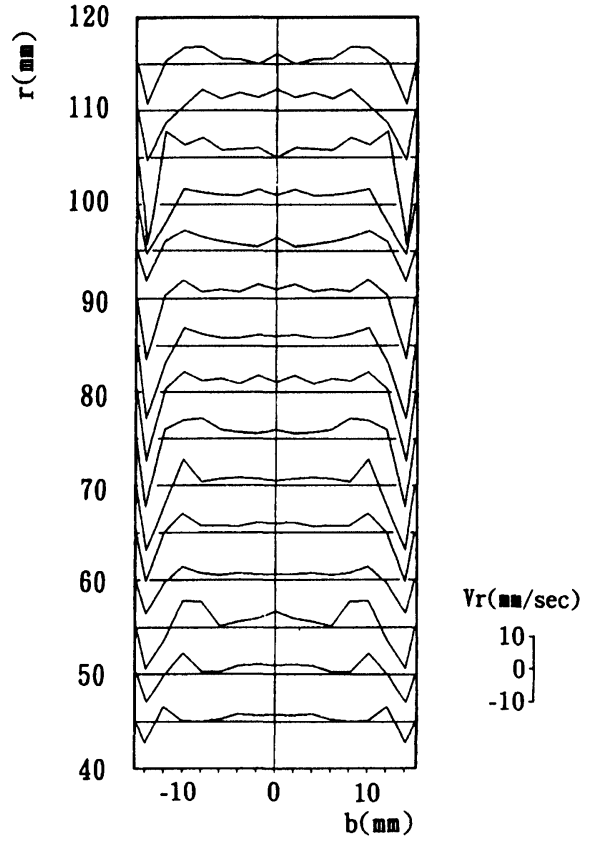

Radial velocity distribution

FIGURE 9(a) Flow pattern and radial velocity distribution on meridional plane for water in Drum II at $6 \mathrm{sec}$ under a spin-down process from 5 sec/rev to zero.

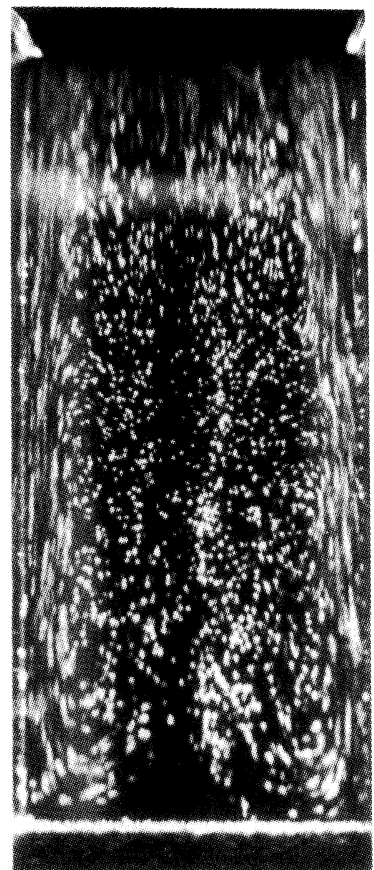

Flow pattern (shutter speed: $1 \mathrm{sec}$ )

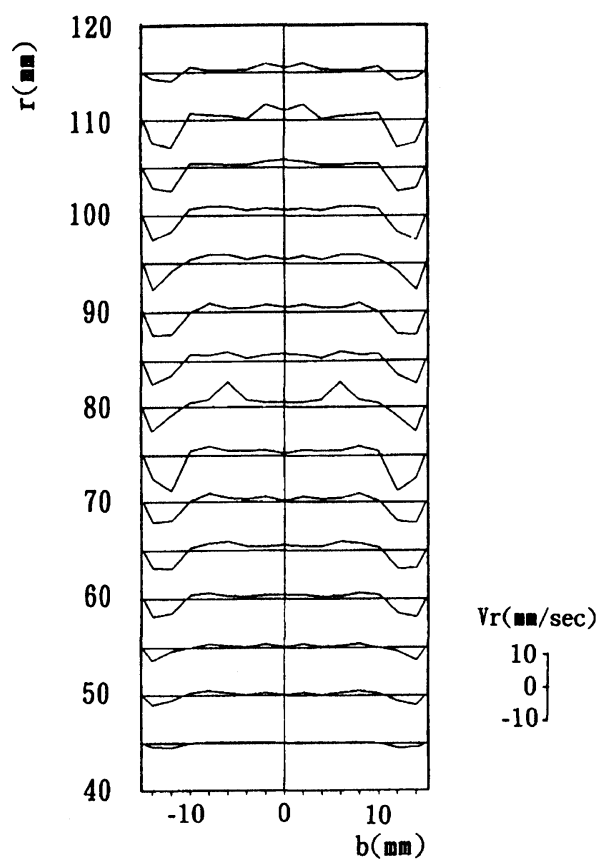

Radial velocity distribution

Figure 9(b) Flow pattern and radial velocity distribution on meridional plane for water in Drum II at 20 sec under a spin-down process from 5 sec/rev to zero. 


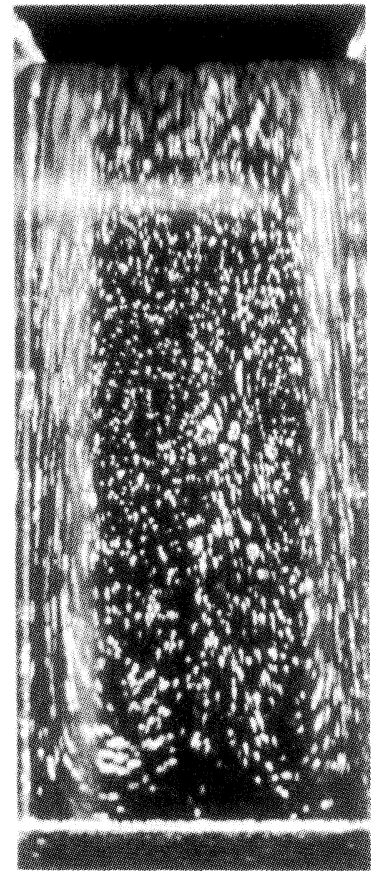

Flow pattern (shutter speed: $1 \mathrm{sec}$ )

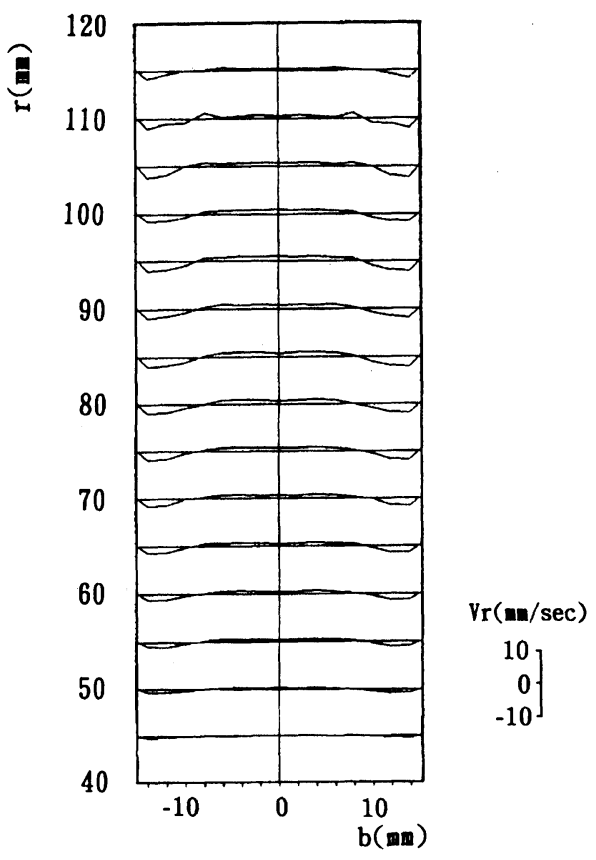

Radial velocity distribution

FIGURE 9(c) Flow pattern and radial velocity distribution on meridional plane for water in Drum II at $40 \mathrm{sec}$ under a spin-down process from $5 \mathrm{sec} / \mathrm{rev}$ to zero.

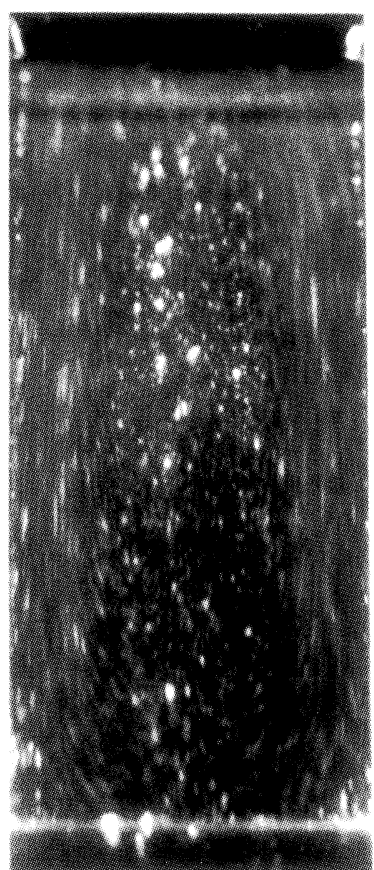

Flow pattern (shutter speed: $2 \mathrm{sec}$ )

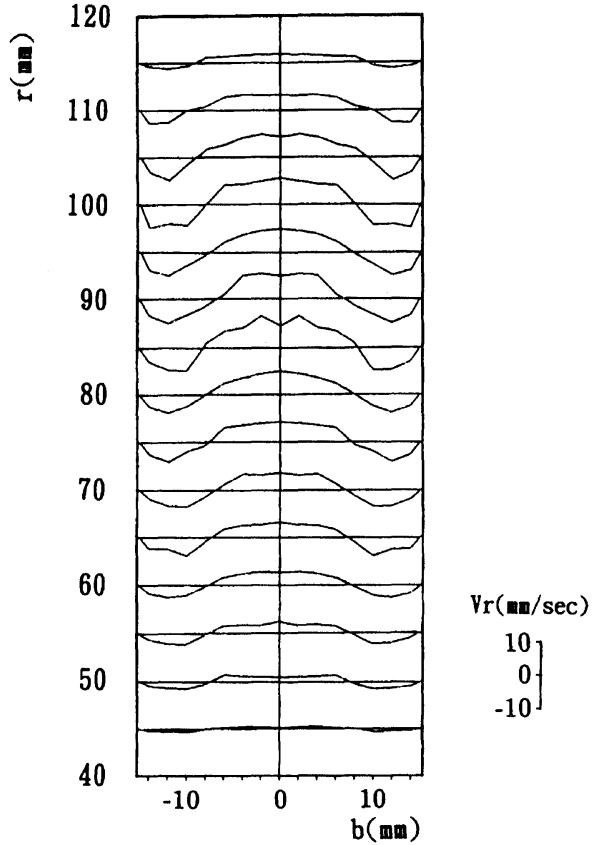

Radial velocity distribution

FIGURE 10(a) Flow pattern and radial velocity distribution on meridional plane for 50\% glycerin/water solution in Drum II at 6 sec under a spin-down process from $5 \mathrm{sec} / \mathrm{rev}$ to zero. 


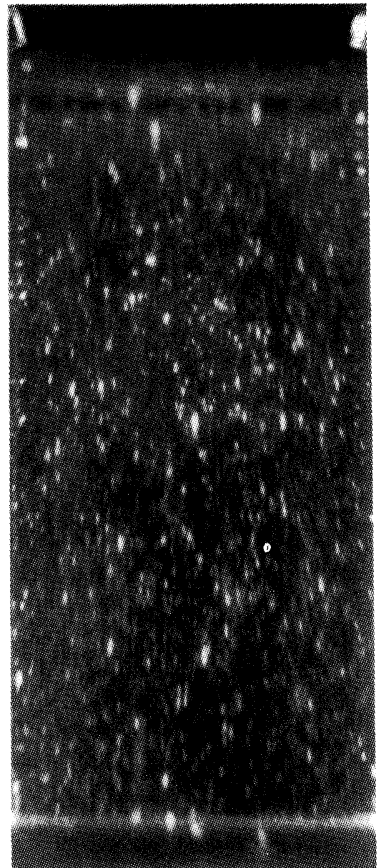

Flow pattern (shutter speed: $1 \mathrm{sec}$ )

FIGURE 10(b) Flow pattern and radial velocity distribution on meridional plane for $50 \%$ glycerin/water solution in Drum II at 20 sec under a

spin-down process from $5 \mathrm{sec} / \mathrm{rev}$ to zero.

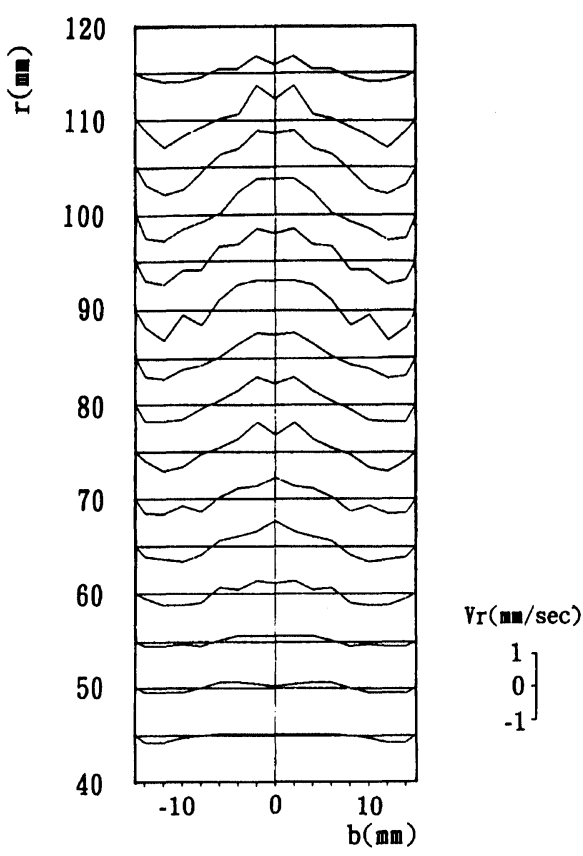

Radial velocity distribution

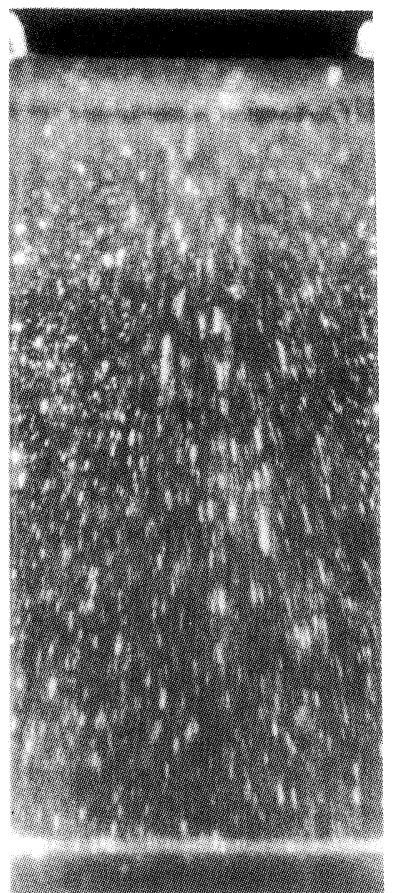

Flow pattern (shutter speed: $4 \mathrm{sec}$ )

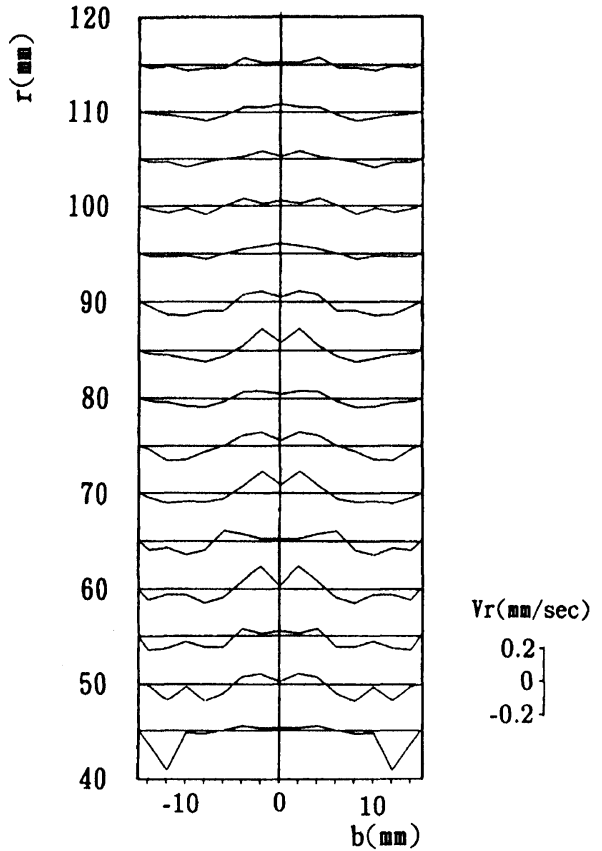

Radial velocity distribution

FIGURE 10 (c) Flow pattern and radial velocity distribution on meridional plane for 50\% glycerin/water solution in Drum II at 40 sec under a spin-down process from $5 \mathrm{sec} / \mathrm{rev}$ to zero. 
between the curved and straight lines in Fig. 7 is caused by the secondary flow.

Figures 9 and 10 show flowpatterns on the meridional plane and their respective radial velocity distributions, Fig. 9 for Drum II, water, $5 \mathrm{sec} / \mathrm{rev}$, Fig. 10 for Drum II, $50 \%$ glycerin/water solution, $5 \mathrm{sec} / \mathrm{rev}$, and (a), (b) and (c) for 6, 20 and $40 \mathrm{sec}$, respectively. Note that Figs. 9 and 10 correspond to Figs. 5 and 6, respectively and that the scale for the radial velocity in Figs. 10(b) and (c) is different from that in other figures. It is.observed in Figs. 9 and 10 that the radial velocity decreases with time with the formation of the Ekman boundary layer on the sidewalls.

Figure 9 depicts a large variation of the radial velocity in the axial direction (right figures). The Ekman boundary layer appeared $1 \mathrm{sec}$ after the initiation of a spindown process (not shown). After $6 \mathrm{sec}$, the radiallyinward velocity in the Ekman boundary layer became higher than the radially-outward velocity in the interior, Fig. 9(a). The fluid in the Ekman boundary layer exited in the axial direction near the inner cylinder, while the fluid near the outer cylinder flowed into the Ekman boundary layer, thus forming a pair of recirculating flows. Each flow exiting from the Ekman boundary layer interacted with the inner cylinder wall to generated a vortex. It is seen in Figs. 9(b) and (c) (right figures) that the radial velocity diminished faster near both the inner and outer cylinder walls than elsewhere due to wall effects. Also observed are a pair of recirculating flows.

In the case of $50 \%$ glycerin/water solution, vortices near the inner cylinder wall were not observed at the initiation of a spin-down process. The radial velocity near the midplane in Fig. 10(a) was larger than that of water shown in Fig. 9(a), while those in Figs. 10(b) and (c) were very small. The decay of the radial velocity was promoted by viscosity.

Figure 11 shows a timewise variation in the thickness of Ekman boundary layer. The thickness in the 50\% glycerin/water solution was larger than that in water due to viscous effect. The flow in the meridional plane can be summarized as follows: The flow in Ekman boundary layer over the sidewall is radially inward. In water, complex vortices appear near the inner cylinder wall. Axial flows near the inner cylinder collided at the midplane. Outside the Ekman boundary layer, a secondary flow is induced toward the outer cylinder, resulting in a symmetrical flow. The thickness of Ekman boundary layer increases with time.

\section{CONCLUSIONS}

1. The appearance of secondary flow result in kinetic energy dissipation to deviate from an exponential

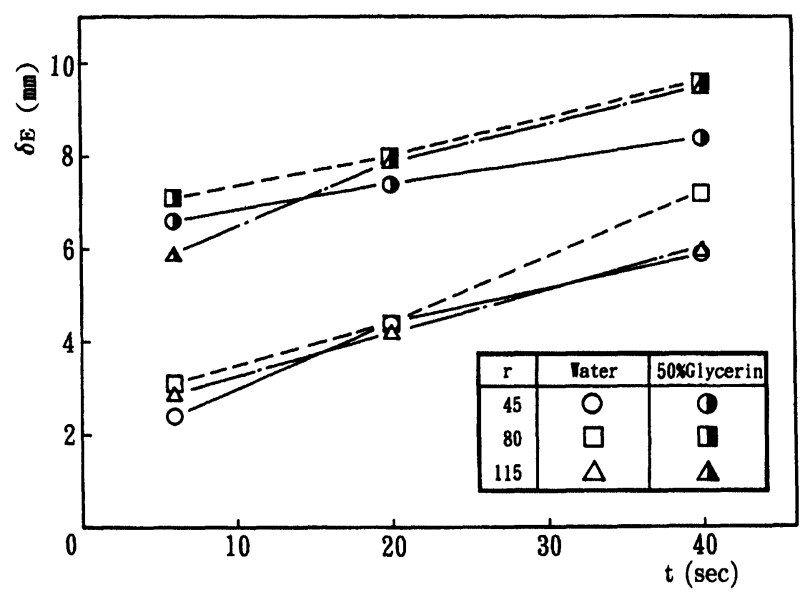

FIGURE 11 Variation of the thickness of Ekman boundary layer with time for Drum II under a spin-down process from $5 \mathrm{sec} / \mathrm{rev}$ to zero.

decay in time, which is caused by a decrease in the angular velocity alone.

2. The formation of Ekman boundary layers on both sidewalls results in a pair of recirculating flows, as seen in flow patterns and also evidenced in radial flow distributions.

3. The thickness of Ekman boundary layer increases with fluid viscosity.

4. The Ekman boundary layer is instrumental in the generation of a pair of recirculating flows inside a rotating drum and reflects a consequence of velocity changes.

\section{References}

Kawashima, G. and Yang, W. J., Unsteady Flow in Rotating Drums using Laser Doppler Velocimetry, Experiments in Fluids, vol. 6, No. 3, pp. 165-171, 1988.

Yang, W. J. and Kawashima, G., Hydrodynamics in Unsteady Rotating Drums, Transport Phenomena in Rotating Machinery, eds. J.H. Kim and W.J. Yang, pp. 51-61, 1990a, Hemisphere Publishing Corporation, Washington, D.C.

Kawashima, G. and Yang, W.J., Light-Sheet Visualization of Unsteady Recirculating Flow inside Rotating Drums, Flow Visualization V, ed. R. Reznicek, pp. 633-639, Hemisphere Publishing Corporation, Washington, D.C., $1990 \mathrm{~b}$.

Kawashima, G. and Yang, W.J., Experimental Study on Unsteady Recirculating Flow in Rotating and Stationary Drum with inner Surface Heating, International Symposium on Nonsteady Fluid Dynamics, eds. J.A. Miller and D.P. Telionis, ASME FED, Vol. 92, pp. 219-224, 1990c.

Yang, W.J. and Kawashima, G., Experimental Study on Unsteady Convection in Rotating Drums with Inner Surface Heating, Preprints of 3rd International Symposium on Transport Phenomena and Dynamics of Rotating Machinery, Honolulu, Vol. 1, pp 156-166, 1990d. 
Kawashima, G and Yang, W.J., Unsteady Recirculating Flow in a Steady Rotating Drum due to Inner Surface Heating, ASME Fluids Engineering-Los Angeles, ASME FED, Vol. 133, pp. 273-274, 1992a.

Yang, W.J., Ohue, H. and Kawashima, G., Experimental Study by LDV and Flow Visualization on the Unsteady Flow Pattern in Rotating Drums, Proceedings of 4th International Symposium on Transport Phenomena and Dynamics of Rotating Machinery, Hawaii, pp. 555-561, 1992b.

Kawashima, G. and Yang, W.J., Determination of Unsteady Flow inside
Rotating Drums by Flow Visualization with Double-Exposure Photographs, ASME-JSME Joint Fluids Engineering Conference, ASME FED, Vol. 106, pp. 41-46, 1991

Kawashima, G., Yang, W.J., and Ohue, H., Image Processing of Flow Patterns in Rotating Drums with Inner Surface Heating, International Seminar on Imaging in Transport Processes, Athens, pp. 197-205, 1992c.

Kawashima, G., Yang, W.J. and Ohue, H., Visualization of Unsteady Flow in Rotating Drums, Flow Visualization VI, ed. Y. Tanida, pp. 62-66, Springer Verlag, Heidelberg, 1992d. 

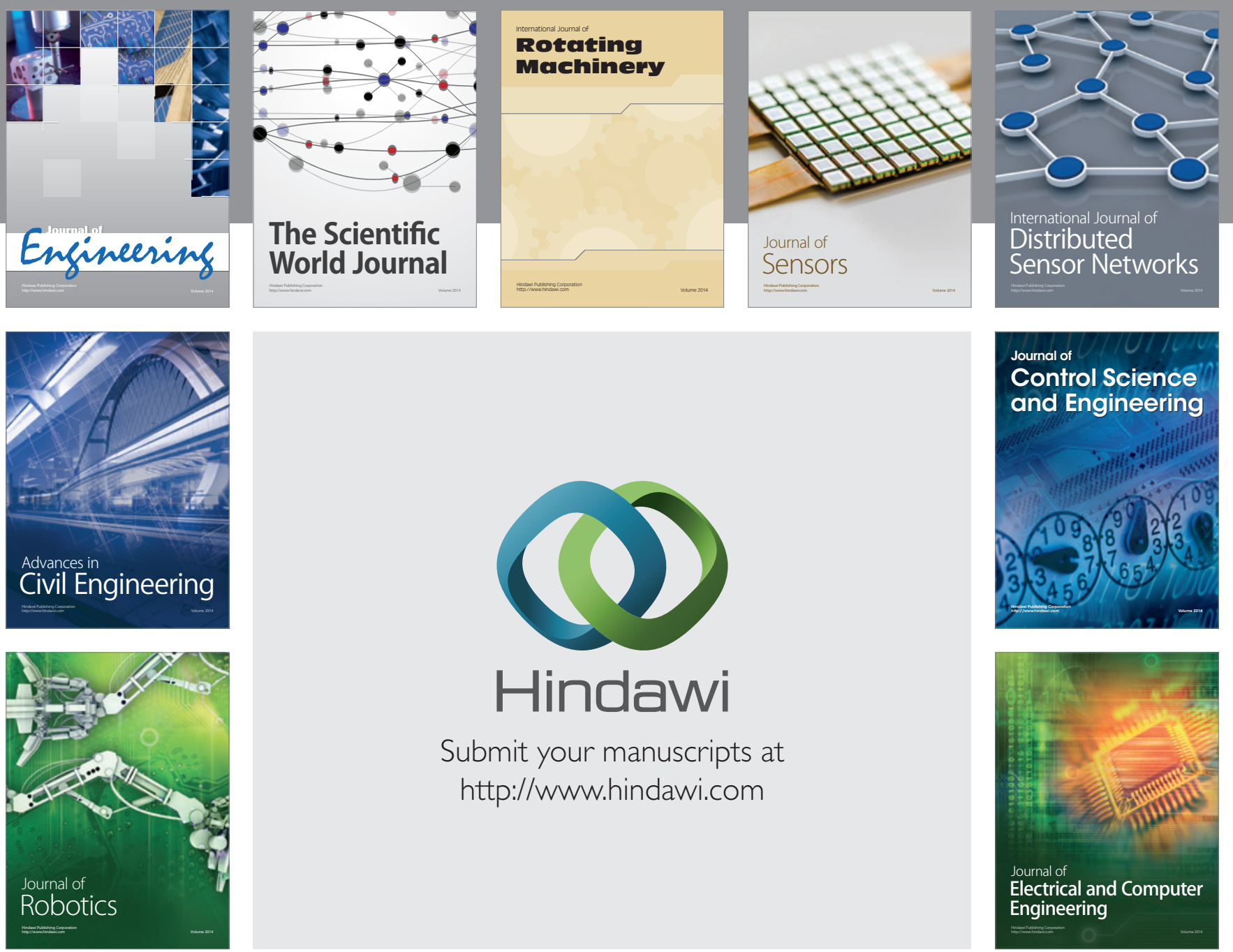

Submit your manuscripts at

http://www.hindawi.com
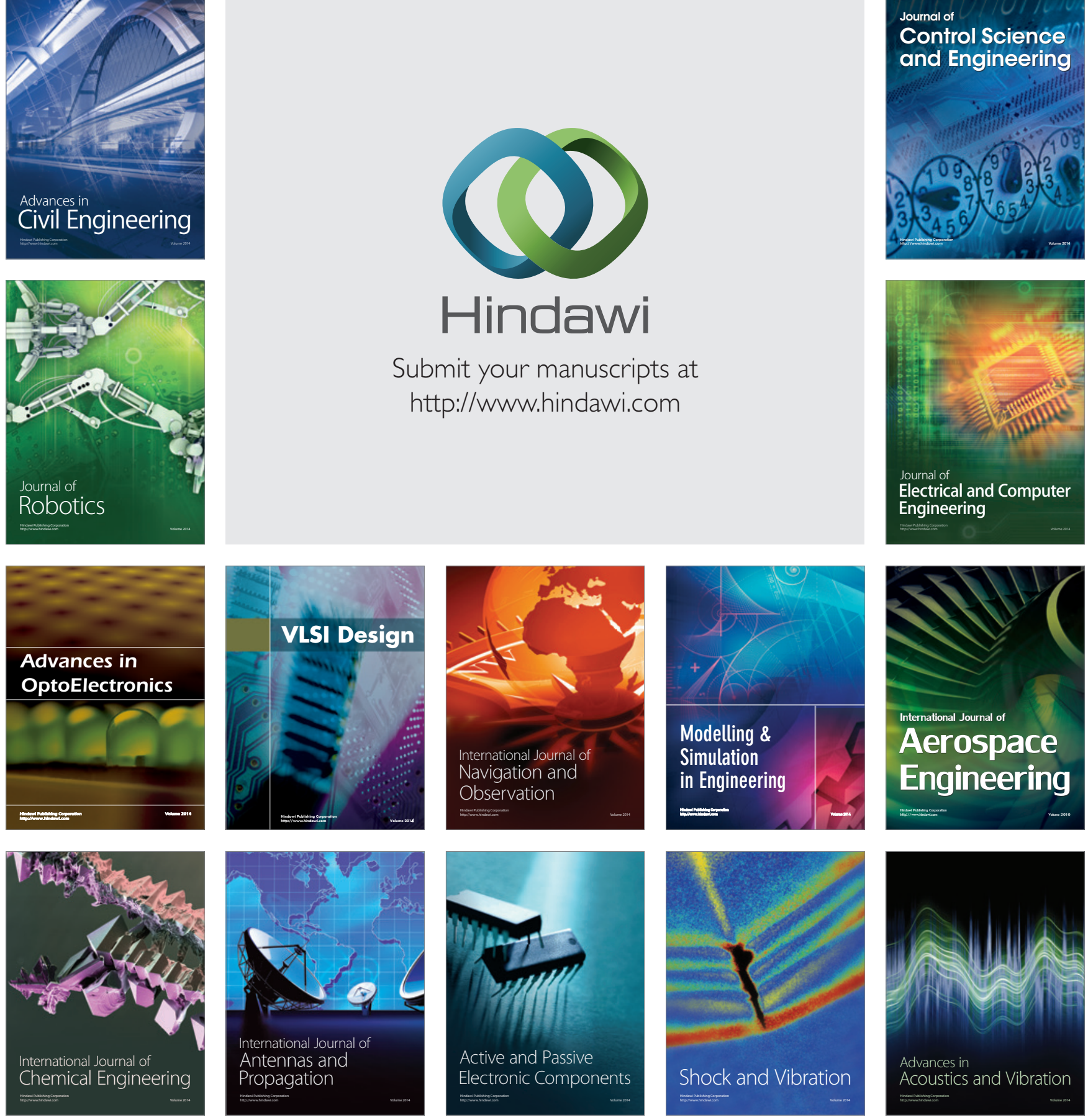